\title{
A comparison of two methods for the diagnosis of lymphogranuloma venereum
}

Correspondence

Sarah Alexander

sarah.alexander@hpa.org.uk

Received 20 January 2008

Accepted 24 April 2008

\author{
Sarah Alexander, Iona M. C. Martin and Catherine Ison
}

Sexually Transmitted Bacteria Reference Laboratory, Health Protection Agency, Centre for Infections, 61 Colindale Ave, London NW9 5HT, UK

\begin{abstract}
A recent outbreak of lymphogranuloma venereum (LGV) within the men who have sex with men (MSM) community and their requirement for extended therapy has highlighted the need for laboratory tests that differentiate LGV-from non-LGV-associated serovars of Chlamydia trachomatis. Two previously described methods were evaluated against 495 clinical specimens referred to the Sexually Transmitted Bacteria Reference Laboratory (London, UK): (i) PCR amplification of a $1.1 \mathrm{~kb}$ region of the omp/ gene followed by restriction enzyme digestion (ompl RFLP-PCR); and (ii) real-time PCR targeting a 36 bp deletion present within the polymorphic membrane protein $\mathrm{H}$ gene of LGV-associated serovars ( $\mathrm{pmpH}$ real-time PCR). For specimens that could be categorized using both methods, a 94.7\% (390/412) concordance was achieved. Eighty-three specimens were found to be untypeable by omp/ RFLP-PCR due to a failure to amplify the $1.1 \mathrm{~kb}$ fragment. Of these 83 untypeable specimens, 19 were determined to be an LGV-associated serovar by $\mathrm{pmpH}$ real-time PCR. Despite the high level of concordance, there were differences found in the technical complexity of the two methods. The $\mathrm{pmpH}$ real-time PCR exhibited greater sensitivity, a more rapid turnaround time and a lower technical requirement. Whilst the omp/ RFLP-PCR was not as robust as a laboratory diagnostic method, it did enable serovar-level identification. LGV infection remains an important threat to the health of high-risk MSM in Europe. In conclusion, the two methods for the detection of LGV from clinical samples were found not only to have a high concordance ( $94.7 \%$ ) but also to be complementary, and could be used in an integrated way to aid LGV detection.
\end{abstract}

\section{INTRODUCTION}

A recent outbreak of lymphogranuloma venereum (LGV) within the men who have sex with men (MSM) community has raised awareness of this disease and highlighted it as a re-emerging public health problem within the UK (Ward et al., 2007). It was previously regarded as a tropical infection (LGV is endemic in parts of Africa, Asia and South America) and, until recently, cases in Europe remained rare and were almost exclusively confined to importation (Jebbari et al., 2007; Ward et al., 2007).

LGV is caused by specific serovars of Chlamydia trachomatis (L1, L2 and L3) and these strains are associated with a more chronic and invasive infection than other serovars. Although LGV symptoms can vary according to site of entry and stage of infection, genital ulceration and inguinal lymphadenopathy are the classical presentations of this disease. However, during the recent European MSM outbreak, anorectal symptoms were the most common presentation, making syndromic management problematic

Abbreviations: LGV, lymphogranuloma venereum; MSM, men who have sex with men; STBRL, Sexually Transmitted Bacteria Reference Laboratory; UNG, uracil glycolase. (van de Laar, 2006; Richardson \& Goldmeier, 2007). LGV infection is also more complex than standard C. trachomatis infection, with patients requiring an extended course of therapy when compared with infection with serovars D$\mathrm{K}$. Because of this, the accurate laboratory diagnosis of LGV serovars of C. trachomatis is essential (BASHH, 2006; McLean et al., 2007).

The laboratory identification of LGV can be problematic, as routine culture of $C$. trachomatis for diagnostic purposes has largely been replaced with nucleic acid amplification techniques; consequently, traditional serotyping methods are now redundant. Two main molecular methods that enable the identification of LGV biovars of C. trachomatis have been described: (i) PCR amplification of a $1.1 \mathrm{~kb}$ region of the outer-membrane I (ompI) gene followed by restriction enzyme digestion (ompI RFLP-PCR; Lan et al., 1993, 1994); and (ii) real-time PCR targeting a $36 \mathrm{bp}$ deletion present within the polymorphic membrane protein $\mathrm{H}(p m p H)$ gene of LGV-associated serovars ( $p m p H$ real-time PCR; Morre et al., 2005).

Clinical specimens referred to the Sexually Transmitted Bacteria Reference Laboratory (STBRL; London, UK) for LGV diagnosis were examined initially using a combina- 
tion of a plasmid-based real-time PCR that detects all $C$. trachomatis serovars and ompI RFLP-PCR. These specimens were then examined retrospectively using the LGVspecific $p m p H$ real-time PCR. Here, we report the first comparative data for the two major methods available for the detection of LGV from clinical specimens.

\section{METHODS}

Specimens examined. A total of 495 specimens from the following sites were examined in this study: rectal (411), urethral (14), other (49) and site unstated (21). All specimens were taken from high-risk symptomatic MSM and were referred to the STBRL during 20042005 for LGV testing.

A range of specimens were received, including fresh dry swabs, unprocessed swabs in transport buffer and residual processed material from nucleic acid amplification techniques. Dry swabs were hydrated with $500 \mu \mathrm{l}$ PBS and agitated on an orbital shaker at 150 r.p.m. for $1 \mathrm{~h}$.

DNA extraction. DNA extractions were performed using one of three methods: (i) manual extraction using a QIAamp Viral RNA Mini kit (Qiagen); (ii) automated extraction using a Corbett DNA X-Tractor with a Macherey-Nagel blood extraction kit; or (iii) MagNA Pure automated extraction (Roche). The three different methods of DNA extraction were employed to cope with increasing levels of specimen referrals, with each representing an advancement in automation. All extractions were performed according to the manufacturer's instructions and each clinical specimen was extracted using only a single DNA extraction method.

C. trachomatis detection. The Chlamydia status of all specimens was determined using the method of Chen et al. (2007). The PCR contained primers targeting an $88 \mathrm{bp}$ region of the $C$. trachomatis cryptic plasmid and primers targeting the human RNase $\mathrm{P}$ gene, which acted as an internal control. All reactions were performed on a Corbett RotorGene-3000. Each $25 \mu$ reaction contained the following primers and reagents: $200 \mathrm{nM}$ CTF-008 (5'-GGATTGACTCCGACAACGTATTC-3'), $300 \mathrm{nM}$ CTR-009 (5'-ATCATTGCCATTAGAAAGGGCATT-3'), 200 nM CTP-010 (5'-FAM-TTACGTGTAGGCGGTTTAGAAAGCGG-BHQ1-3'), 80 nM RNPF-003 (5'-AGATTTGGACCTGCGAGCG-3'), $80 \mathrm{nM}$ RNPR-002 (5'-GAGCGGCTGTCTCCACAAGT-3'), $80 \mathrm{nM}$ RNPP-001 (5'-CY5-TTCTGACCTGAAGGCTCTGCGCG-BHQ3-3'), $200 \mu \mathrm{M}$ dATP, $200 \mu \mathrm{M}$ dCTP, $200 \mu \mathrm{M}$ dGTP, $400 \mu \mathrm{M}$ dUTP, $1 \times$ PCR buffer (Applied Biosystems), $4 \mathrm{mM} \mathrm{MgCl}_{2}, 5 \mathrm{U}$ AmpliTaq Gold polymerase (Applied Biosystems), $0.5 \mathrm{U}$ uracil glycolase (UNG) and $10 \mu \mathrm{DNA}$ template. Reaction conditions were: $2 \mathrm{~min}$ at $50{ }^{\circ} \mathrm{C}$ (denaturation of UNG) and $10 \mathrm{~min}$ at $95^{\circ} \mathrm{C}$ (hot-start Taq activation), followed by 45 cycles of $25 \mathrm{~s}$ at $95{ }^{\circ} \mathrm{C}$ (denaturation) and $1 \mathrm{~min}$ at $60{ }^{\circ} \mathrm{C}$ (combined annealing and extension). In instances where the specimens were found to be negative for $C$. trachomatis, their negative status was confirmed using an Artus C. trachomatis Real-Art kit. In instances where the Artus kit and the in-house plasmid-based PCR produced positive results after 40 cycles, the specimen was described as equivocal.

Genotyping methods by RFLP. Genotyping of the samples positive for C. trachomatis was performed using the methods of Lan et al. (1993, 1994). Briefly, a $1.1 \mathrm{~kb}$ section of the ompI gene of $C$. trachomatis was amplified by nested PCR. The first round contained primers NLO (5'-ATGAAAAAACTCTTGAAATCG-3') and NRO (5'-CTCAACTGTAACTGCGTATTT-3'). The second round contained primers PCTM3 (5'-TCCTTGCAAGCTCTGCCTGTGGGGAATCCT- $\left.3^{\prime}\right)$ and CT4 (5'-CCGCAAGATTTTCTAGATTTCATC-
TTGT- $\left.3^{\prime}\right)$. All PCR runs contained positive controls for serovars L1, L2 and L3 and a negative control. Amplification was confirmed using a DNA7500 LabChip on an Agilent Bioanalyser. Products were then digested for $2 \mathrm{~h}$ with $A l u \mathrm{I}$ at $37^{\circ} \mathrm{C}$ and separated on a DNA7500 LabChip. Digestion patterns were compared with those obtained for controls L1, L2 and L3. Samples with a restriction digest pattern giving an L3 profile were additionally digested using EcoRI and DdeI at $37^{\circ} \mathrm{C}$ for $2 \mathrm{~h}$ to differentiate between serovars $\mathrm{H}$, I and L3. Samples were assigned as being LGV-associated or non-LGV-associated on the basis of their restriction digest profile.

pmpH real-time PCR. The LGV-specific $p m p H$ real-time PCR assay was performed retrospectively on specimens that had been examined previously by ompI RFLP-PCR. The assay was carried out on a RotorGene 3000 PCR instrument according to the methods of Morre et al. (2005). Each $20 \mu \mathrm{l}$ reaction contained the following: $250 \mathrm{nM} \mathrm{LGV-F}$ (5'-CTGTGCCAACCTCATCATCAA-3'), $250 \mathrm{nM}$ LGV-R $\quad\left(5^{\prime}-\right.$ AGACCCTTTCCGAGCATCACT-3'), $500 \mathrm{nM}$ LGV-Probe (5'-6FAM-CCTGCTCCAACAGT-MGB-3' $), \quad 200 \mu \mathrm{M} \quad$ dATP, $200 \mu \mathrm{M}$ dCTP, $200 \mu \mathrm{M}$ dGTP, $400 \mu \mathrm{M}$ dUTP, $1 \times$ PCR buffer (Applied Biosystems), $4 \mathrm{mM} \mathrm{MgCl}_{2}, 5 \mathrm{U}$ AmpliTaq Gold polymerase, $0.5 \mathrm{U}$ UNG and $5 \mu \mathrm{l}$ DNA template. Reaction conditions were: $2 \mathrm{~min}$ at $50{ }^{\circ} \mathrm{C}$ (denaturation of UNG) and $10 \mathrm{~min}$ at $95{ }^{\circ} \mathrm{C}$ (hot-start Taq activation), followed by 45 cycles of $15 \mathrm{~s}$ at $95{ }^{\circ} \mathrm{C}$ (denaturation) and $1 \mathrm{~min}$ at $60{ }^{\circ} \mathrm{C}$ (combined annealing and extension).

Where ompI RFLP-PCR and $p m p H$ real-time PCR produced discordant results, both tests were repeated, if sufficient sample was available, to confirm the results in an attempt to resolve the discordant status.

\section{RESULTS}

\section{Comparison of ompl RFLP-PCR and $p m p H$ real- time PCR}

A total of 495 specimens sourced from high-risk patients were examined using both a combination of a $C$. trachomatis-specific in-house PCR and ompI RFLP-PCR, and a $p m p H$ LGV-specific real-time PCR assay (Table 1).

Table 1. Evaluation of the two different methods for the detection of LGV from clinical samples

Results are given as the number of samples. Three specimens produced $p m p H$ real-time PCR discordant results that could not be resolved and therefore are not represented in Table 1, giving a total of 492 samples. An explanation of these three specimens is given in Results.

\begin{tabular}{|lcc|}
\hline \multirow{2}{*}{\begin{tabular}{l} 
Results of $\begin{array}{c}\text { ompI RFLP-PCR } \\
\text { and C. trachomatis plasmid } \\
\text { real-time PCR confirmation }\end{array}$ \\
\cline { 2 - 3 }
\end{tabular}} & \multicolumn{2}{c|}{$\boldsymbol{p m p H}$ real-time PCR } \\
\cline { 2 - 3 } & Positive & Negative \\
\hline LGV & 163 & 5 \\
Non-LGV associated & 14 & 141 \\
ompI failed to amplify & 19 & 64 \\
Negative & 0 & 78 \\
Equivocal & 0 & 6 \\
Inhibited & 0 & 2 \\
\hline
\end{tabular}


The two methods produced concordant results in 390 of the specimens examined: (i) 163 specimens were determined to be LGV using both methods; and (ii) 227 specimens were determined to be negative using the $p m p H$ real-time PCR, of which 78 specimens were negative for $C$. trachomatis, six were equivocal for $C$. trachomatis and 141 were determined to be non-LGV-associated by ompI RFLP-PCR typing. Two specimens were found to be PCR inhibitory. Eighty-three specimens (which were determined to be C. trachomatispositive using the plasmid-based real-time PCR) were untypeable using the ompI RFLP-PCR assay due to repeated failure to amplify the $1.1 \mathrm{~kb}$ fragment. Of the 83 untypeable specimens by ompI RFLP-PCR, 19 were found to be positive for LGV using the $p m p H$ real-time PCR. This highlights the increased sensitivity that can be achieved when using a realtime PCR-based method. Ultimately, for specimens that could be categorized using both methods, a $94.7 \%$ (390/ 412) concordance was achieved.

Twenty-two specimens (22/412;5.3\%) were found to be discordant between the two methods. Five of these were negative by $p m p H$ real-time PCR but generated profiles that were consistent with L2 by ompI RFLP-PCR. The retrospective analysis of the specimen collection using pmpH real-time PCR is a plausible explanation for these discordant specimens, as DNA degradation could have occurred. However, it is also possible that some of these specimens were initially misidentified as LGV serovars by ompI RFLP-PCR due to the subjective and complex nature of the interpretation of the banding profiles. In our laboratory, only LGV serovars (L1, L2 and L3) are included as positive controls, rather than a full set of all $14 \mathrm{C}$. trachomatis serovars. This can sometimes make the interpretation of ompI RFLP-PCR profiles difficult, as some digests of standard serovars of $C$. trachomatis can closely resemble those of LGV strains.

Fourteen specimens were found to be positive using the $p m p H$ real-time PCR method but non-LGV-associated using ompI RFLP-PCR. It is unlikely that false-positive $p m p H$ real-time PCR results can explain these discrepancies, as repeat testing of ten of these specimens confirmed that these samples were reproducibly positive by $p m p H$ real-time PCR (the four remaining specimens had insufficient volume for repeat testing). It should be noted that the specimens examined in this study were obtained from very high-risk patients and therefore the possibility of mixed-serovar $C$. trachomatis infections cannot be overlooked. This could provide a highly likely explanation for these discrepant results, as the real-time PCR method only requires one LGV $p m p H$ target to generate a positive result, whereas the ompI RFLP-PCR would generate either a mixed profile or a profile of the most abundant $C$. trachomatis serovar, which could have resulted in a result for non-LGV-associated serovar of $C$. trachomatis being generated. Again, it is also possible that the subjective nature of RFLP-PCR profiles could have resulted in some weak or partially digested LGV profiles being misidentified as non-LGV-associated serovars.
The remaining three clinical specimens produced discrepant results when examined by $p m p H$ real-time PCR and these could not be resolved. For all three specimens, two DNA extractions were performed from the original swab transport medium because the ompI gene had failed to amplify in the initial RFLP-PCR. When retrospectively performing the $p m p H$ real-time PCR on both extractions from all three specimens, discordant results were produced, with one extraction producing a positive result and one producing a negative result. Attempts were made to resolve the discordant status of two of these specimens by performing a fresh DNA extraction (one specimen had insufficient volume for a repeat extraction); however, both specimens had completely degraded.

\section{DISCUSSION}

The recent outbreak of LGV throughout western Europe has highlighted the need for patients infected with $\mathrm{L}$ serovars of $C$. trachomatis to be given extended antimicrobial therapy and the problems associated with trying to diagnosis this condition syndromically. Consequently, rapid and reliable laboratory methods that can differentiate LGV- from non-LGV-associated C. trachomatis infections are extremely important. In this paper, we provide the first comparative data for the two major methods available for the diagnosis of LGV.

This study compared two methods for the detection of LGV from clinical samples. Whilst both methods showed high levels of concordance ( $94.7 \%$ ), they were found to differ in terms of technical complexity. The greater sensitivity, shorter turnaround time and lower technical requirement of the $p m p H$ real-time PCR test would make it the test of choice for diagnostic use. The ompI RFLP-PCR is not a diagnostic test but rather a $C$. trachomatis genotyping method, which enables serovar-level identification of a specific clinical specimen. However, this method lacks sensitivity, has a lengthy turnaround time and requires both experienced staff and specialist equipment, and therefore is almost exclusively confined to specialized centres. In the UK, all LGV infection is detected at a central facility (STBRL), where currently the LGV testing algorithm is to test any referred specimen for LGV using the more sensitive and rapid $p m p H$ real-time PCR. All $p m p H$ real-time PCRpositive specimens are then examined further using the $\mathrm{ompI}$ RFLP-PCR to identify the precise serovar of LGV responsible and to confirm the original $p m p H$ result.

Ultimately, the detection of a LGV-positive clinical specimen using either of the methods examined in this study will always be technically demanding, as specimens first require a primary screen with a $C$. trachomatis diagnostic test. Such an approach has been problematic historically, as not all commercial C. trachomatis platforms are approved by the Food and Drug Administration for the examination of rectal specimens, although recent data suggest that these platforms exhibit high specificity when 
presented with these specimens (Alexander et al., 2007). In addition, whilst a recent real-time PCR method has been described that enables the simultaneous detection of both C. trachomatis serovars and L2 strains specifically, such a method would clearly miss LGV infections caused by L1 and L3 serovars (Halse et al., 2006).

LGV infection remains an important threat to the health of high-risk MSM in Europe, with 492 cases of LGV confirmed within the UK to date (Jebbari et al., 2007). In the UK - unlike other European countries - the case definition of a confirmed LGV infection is one that has been detected in the laboratory using solely molecular methods. In this study, two methods were compared for the detection of LGV from clinical samples, both of which were found not only to have a high concordance $(94.7 \%)$, but also to be complementary, and could be used in an integrated way to aid LGV detection.

\section{ACKNOWLEDGEMENTS}

Authors would like to thank the microbiologists and GUM staff who referred specimens to STBRL for LGV detection and all members of the LGV UK Incident Group.

\section{REFERENCES}

Alexander, S., Martin, I. \& Ison, C. (2007). Confirming the Chlamydia trachomatis status of referred rectal specimens. Sex Transm Infect 83, 327-329.

BASHH (2006). National Guideline for the Management of Lymphogranuloma venereum ( $L G V$ ). Available at: http://www.bashh. org/guidelines/2007/lgv_gdl_revised_final2_1106.pdf.
Chen, C. Y., Chi, K. H., Alexander, S., Martin, I. M., Liu, H., Ison, C. A. \& Ballard, R. C. (2007). The molecular diagnosis of lymphogranuloma venereum: evaluation of a real-time multiplex polymerase chain reaction test using rectal and urethral specimens. Sex Transm Dis 34, 451-455.

Halse, T. A., Musser, K. A. \& Limberger, R. J. (2006). A multiplexed real-time PCR assay for rapid detection of Chlamydia trachomatis and identification of serovar L-2, the major cause of lymphogranuloma venereum in New York. Mol Cell Probes 20, 290-297.

Jebbari, H., Alexander, S., Ward, H., Evans, B., Solomou, M., Thornton, A., Deans, G., White, J., French, P. D. \& Ison, C. A. (2007). Update on lymphogranuloma venereum in the UK. Sex Transm Infect 83, 324-326.

Lan, J., Walboomers, J. M. M., Roosendaal, R., Doornum, G. J. J., Maclaren, D. M., Meijer, C. J. L. M. \& Brule, A. J. C. (1993). Direct detection and genotyping of Chlamydia trachomatis in cervical scrapes by using polymerase chain reaction and restriction fragment length polymorphism analysis. J Clin Microbiol 31, 1060-1065.

Lan, J., Ossewaarde, J. M., Walboomers, J. M. M., Meijer, C. J. L. M. \& Brule, A. J. C. (1994). Improved PCR sensitivity for direct genotyping of Chlamydia trachomatis serovars by using a nested PCR. J Clin Microbiol 32, 528-530.

McLean, C. A., Stoner, B. P. \& Workowski, K. A. (2007). Treatment of lymphogranuloma venereum. Clin Infect Dis 44 (Suppl. 3), S147-S152.

Morre, S. A., Spaargaren, J., Fennema, J. S., de Vries, H. J., Coutinho, R. A. \& Pena, A. S. (2005). Real-time polymerase chain reaction to diagnose lymphogranuloma venereum. Emerg Infect Dis 11, 1311-1312.

Richardson, D. \& Goldmeier, D. (2007). Lymphogranuloma venereum: an emerging cause of proctitis in men who have sex with men. Int J STD AIDS 18, 11-14.

van de Laar, M. J. (2006). The emergence of LGV in western Europe: what do we know, what can we do? Euro Surveill 11, 146-148.

Ward, H., Martin, I., Macdonald, N., Alexander, S., Simms, I., Fenton, K., French, P., Dean, G. \& Ison, C. (2007). Lymphogranuloma venereum in the United Kingdom. Clin Infect Dis 44, 26-32. 